\title{
Rare taxa maintain the stability of crop mycobiomes and ecosystem
}

\section{functions}

Chao Xiong ${ }^{1,2}$, Ji-Zheng He ${ }^{1,2}$, Brajesh K. Singh ${ }^{3,4}$, Yong-Guan Zhu ${ }^{1,5}$, Jun-Tao Wang ${ }^{1}$, Pei-Pei Li ${ }^{6}$, Qin-Bing Zhang ${ }^{7}$, Li-Li Han ${ }^{1,2}$, Ju-Pei Shen ${ }^{1,2}$, An-Hui Ge ${ }^{1,2}$, Chuan-Fa $\mathrm{Wu}^{1,6}$, Li-Mei Zhang ${ }^{1,2 *}$

${ }^{1}$ State Key Laboratory of Urban and Regional Ecology, Research Center for Eco-Environmental Sciences, Chinese Academy of Sciences, Beijing 100085, China

${ }^{2}$ University of Chinese Academy of Sciences, Beijing 100049, China

${ }^{3}$ Global Centre for Land-Based Innovation, Western Sydney University, Penrith South DC, NSW 2751, Australia

${ }^{4}$ Hawkesbury Institute for the Environment, Western Sydney University, Penrith, New South Wales 2751, Australia

${ }^{5}$ Key Laboratory of Urban Environment and Health, Institute of Urban Environment, Chinese Academy of Sciences, Xiamen 361021, China

${ }^{6}$ College of Resource and Environmental Sciences, Henan Agricultural University, Zhengzhou 450002, China

${ }^{7}$ Soil and Fertilizer Station of Qilin District, Qujing, Yunnan Province, Qujing, 655000, China

This is the author manuscript accepted for publication and has undergone full peer review but has not been through the copyediting, typesetting, pagination and proofreading process, which may lead to differences between this version and the Version of Record. Please cite this article as doi: 10.1111/1462-2920.15262

This article is protected by copyright. All rights reserved. 
*Correspondence: zhanglm@rcees.ac.cn (Li-Mei Zhang)

Running title: Rare taxa play a key role in crop mycobiomes

\section{Originality-Significance Statement}

Highly diverse microbes inhabit every plant compartment, including rhizosphere, root, stem, leaf, etc., and act as a secondary genome of the plant. The plant-associated fungal communities (mycobiomes) play a crucial role in nutrient acquisition, plant health, host fitness and plant productivity. Here we examined the community assembly of mycobiomes in different compartments (across soil, root and leaf) of three crops under different fertilization practices at two contrasting sites. Our results demonstrate that rare fungal taxa play a central role in sustaining the stability of crop mycobiomes network and ecosystem functions, and that crop mycobiomes are shaped predominantly by host selection rather than by environmental factors. This study provides a holistic understanding on the ecological processes that underpin the assembly of plant mycobiomes along the soil-plant continuum and advances our knowledge on the plant-microbes interactions, which have important implications for future manipulation of crop microbiome for sustainable agriculture. 


\section{Summary}

Plants harbor highly diverse mycobiomes which sustain essential functions for host health and productivity. However, ecological processes that govern the plant-mycobiome assembly, interactions and their impact on ecosystem functions remain poorly known. Here we characterized the ecological role and community assembly of both abundant and rare fungal taxa along the soil-plant continuums (rhizosphere, phyllosphere and endosphere) in the maize-wheat/barley rotation system under different fertilization practices at two contrasting sites. Our results indicate that mycobiome assembly is shaped predominantly by compartment niche and host species rather than by environmental factors. Moreover, crop-associated fungal communities are dominated by few abundant taxa (mainly belonging to Sordariomycetes and Dothideomycetes), while the majority of diversity within mycobiomes are represented by rare taxa. For plant compartments, the abundant sub-community is mainly determined by stochastic processes. In contrast, the rare sub-community is more sensitive to host selection and mainly governed by deterministic processes.

Further, our results demonstrate that rare taxa play an important role in fungal co-occurrence network and ecosystem functioning like crop yield and soil enzyme activities. These results significantly advance our understanding of crop mycobiome assembly and highlight the key role of rare taxa in sustaining the stability of crop mycobiomes and ecosystem functions.

Keywords: fungal community, plant-microbe interactions, community assembly, abundant taxa, 
rare taxa, soil-plant continuum

\section{Introduction}

Plants harbor highly diverse microbiomes and co-evolve with them since their origin in terrestrial ecosystems (Bulgarelli et al., 2013; Muller et al., 2016). The plant-associated fungal communities (mycobiomes) play a crucial role in plant productivity, nutrient acquisition, tolerance of biotic and abiotic stresses, and plant-soil feedbacks (Lau \& Lennon, 2011; Hacquard et al., 2015; Vandenkoornhuyse et al., 2015; Martin et al., 2017). Understanding the mechanisms that underpin the host microbiome assembly is critical for the development of future sustainable agriculture tools. Significant efforts have therefore been made to identify the drivers and mechanisms of microbiome assembly but mainly focused on bacteria and rhizosphere samples (Bulgarelli et al., 2015; Edwards et al., 2015). In addition, most studies on fungal community focused on mycorrhizal fungi, while there is growing evidence that other fungal taxa also play significant roles in plant fitness (Almario et al., 2017; Mommer et al., 2018; Morcillo et al., 2020). For example, it was reported that nonmycorrhizal taxa like Helotiales is able to promote plant growth and $\mathrm{P}$ uptake under P-limited conditions (Almario et al., 2017). Despite the importance, we have little knowledge on the ecological processes that govern the plant mycobiome assembly, particularly for epiphytic and endophytic fungi of leaves and roots (Vorholt, 2012; Vandenkoornhuyse et al., 2015; Singh et al., 2020). Uncovering how the plant host shapes 
fungal communities and how these complex fungal communities respond to different agronomic managements are the first step to develop effective tools to increase plant productivity in a sustainable way.

The eco-evolutionary theory suggests that microbial communities in most habitats normally show a skewed species abundance distribution, in which a few of species (i.e. abundant taxa) dominate the community while a large number of species (i.e. rare taxa) exist with very low abundance and are often ignored (Pedros-Alio, 2012; Lynch \& Neufeld, 2015). The increasing evidence indicates that rare taxa play important roles in driving ecosystem functions and have a different response to environmental changes from abundant taxa (Jiao et al., 2017; Jousset et al., 2017; Ziegler et al., 2018). For example, studies on coral-algal assemblages suggested that the rare taxa play a key role in co-occurrence network and may greatly contribute to the stability of the host-symbiont community by replacing dominant ones under environmental stress (Boulotte et al., 2016; Ziegler et al., 2018). Moreover, it has been widely accepted that microbiome assembly under various conditions can be explained by niche theory or neutral theory, of which niche theory considers that biotic and abiotic factors determine microbial communities (i.e. deterministic processes). In contrast, neutral theory regards that microbial communities are mainly shaped by random changes, including random birth, death, and dispersal events (i.e. stochastic processes) (Zhou et al., 2014). Whereas, some recent studies suggested that the two processes have different effects on rare and abundant sub-communities (Liu et al., 2015; Jiao et al., 2017; 
Gao et al., 2020). For instance, a recent work on agricultural soils reported that rare sub-community is dominated by deterministic processes while abundant sub-community is influenced largely by stochastic processes (Jiao \& Lu, 2020). Therefore, removing rare taxa from a dataset may neglect those rare but keystone species and may hinder our understanding of the microbiome assembly, plant-microbiome interactions and host fitness (Mouillot et al., 2013; Jousset et al., 2017; Toju et al., 2018).

The plant performance depends on the complex and dynamic interactions among host plant, microbiome and environment, with multiple relationships including "microbes-plant", “plant-microbes”, and “plant-microbes-environment” (Muller et al., 2016; Foster et al., 2017; Martin et al., 2017). Among these, the "microbes to plant" relationships have been widely investigated, and provided strong evidences that fungal communities not only affect the plant nutrient acquisition and productivity, but also influence the distribution and population dynamics of plant community (Lau \& Lennon, 2011; Almario et al., 2017; Semchenko et al., 2018). More recently, some studies explored "host to microbes" relationship and suggested the critical role of host in shaping plant mycobiomes (Hamonts et al., 2017; Cregger et al., 2018; Pent et al., 2018; Gao et al., 2020). For example, studies on mycobiomes in roots and aboveground compartments of sorghum (Gao et al., 2020) and sugarcane (Hamonts et al., 2017) have suggested that different plant compartments (e.g. root, stem and leaf) harbor distinct fungal communities, and that host genotype (i.e. different cultivars) has a significant effect on root fungal communities but not on 
leaf fungal communities (Gao et al., 2020). A work on fungal communities in leaves of mangrove showed that plant species has a greater effect on endophytic fungi than on epiphytic fungi (Yao et al., 2019). However, we know little about the interactions among plant, microbes and environment. Particularly, a systematic understanding of how host and environmental factors shape the assembly and co-occurrence patterns of plant mycobiomes, and how abundant and rare taxa respond to these factors is still scarce.

Multiple practices such as straw retention (Sun et al., 2015), application of nitrification inhibitor (Wu et al., 2018), biochar amendment (Edwards et al., 2018), and transplantation of beneficial microbes (Van der Heijden et al., 1998; Christian et al., 2019) have been employed to improve soil fertility and reduce the use of chemical $\mathrm{N}$ fertilization. However, there are large knowledge gaps on how these practices influence the assemblies and functions of mycobiomes along the soil-plant continuum, and how abundant and rare sub-communities respond to these fertilization practices. Here, the fungal communities were investigated across 468 samples collected from bulk soil, rhizosphere and 4 plant compartment niches (rhizoplane, root endosphere, phylloplane and leaf endosphere) in the maize-wheat/barley rotation system under different fertilization practices (e.g. Control without $\mathrm{N}$ fertilization, Local habit fertilization and $20 \% \mathrm{~N}$ reduction treatments etc.) at two geographically distinct and contrasting sites (e.g. with different soil types and $\mathrm{pH}$ ). Our aims were to (1) identify the relative contribution of host and environmental factors in shaping crop mycobiomes along the soil-plant continuum, and assessed 
how deterministic and stochastic processes act on abundant and rare sub-communities; (2) explore the species traits, ecological functions, and co-occurrence patterns of abundant and rare fungal taxa. We hypothesized that (1) crop mycobiomes are greatly shaped by host selection (i.e. compartment niche and host species) rather than by environmental factors, and rare taxa may be more sensitive to host selection and mainly governed by deterministic processes; (2) rare taxa may play a central role in maintaining the stability of crop mycobiomes and ecosystem functions as they represent the majority of microbial diversity and are functionally redundant.

\section{Results}

\section{Taxonomic composition and functional guilds of mycobiomes along the soil-plant continuum}

Our results showed that fungal communities along the soil-plant continuum were dominated by few abundant taxa with wide niche breadth, with the remaining rare taxa highly specific in different habitats (Fig. 1). Among the total 7,699 zero-radius operational taxonomic units (ZOTUs), $1.8 \%$ of ZOTUs (142) were classified as abundant taxa (ZOTUs with a relative abundances $\geq 1 \%$ within a sample and $\geq 0.1 \%$ across all sample) and accounted for $77.0 \%$ of total sequences. In contrast, $70.1 \%$ of ZOTUs (5397) were classified as rare taxa (ZOTUs with a relative abundance $<0.01 \%$ within a sample and $<0.001 \%$ across all samples) but just accounted for $1.5 \%$ of total sequences (Fig. 1a, Table S1). Phylogenetic relationships within abundant taxa were much closer than those within rare taxa, corresponding to a lower phylogenetic diversity 
within abundant taxa (Fig. 1a, c). Coincidently, rare taxa had significantly higher community dissimilarity than abundant taxa, while abundant taxa had greater values of Levin’s niche breadth index $(40.2 \pm 29.8)$ than rare taxa $(11.0 \pm 9.8)$ (Fig. 1c). Furthermore, the relative abundance of individual ZOTU was positively related with its Levin's niche breadth index (Spearman $R^{2}=0.37$, $P<0.001$, Fig. 1c). Fungal community composition and functional guilds differed between abundant and rare taxa, and among different compartment niches (Fig. S1a, b). Both abundant and rare sub-communities were dominated by phylum Ascomycota, with Sordariomycetes and Dothideomycetes classes accounting for more than 50\% of total sequences (Fig. 1a-b, Fig. S1a). Remarkably, the class Glomeromycetes (Arbuscular mycorrhiza fungi, AMF) was exclusively present in rare sub-community (Fig. 1a-b, Fig. S1a). Fungal functional guilds prediction further suggested a relatively higher proportion of group "Saprotroph" and "Symbiotroph" in rare sub-community than in abundant sub-community (Fig. 1b). For different compartment niches, the class Dothideomycetes (29.0\%) and Sordariomycetes (43.7\%) were the most abundant classes in plant niches (leaves and roots) and soils (bulk and rhizosphere), respectively (Fig. S1a). Strikingly, the class Pucciniomycetes (35.3\%) in leaf endosphere and the class Glomeromycetes (AM fungi, $0.4 \%)$ in root endosphere were significantly more abundant than any other compartment niches ( $p$ $<$ 0.001; Fig. S1a, Fig. S2). Coincidently, leaf endosphere possessed more abundant "Plant Pathogen” functional group and root endosphere possessed more "Arbuscular Mycorrhizal” group than any other compartment niches $(p<0.001)$ (Fig. S3). Pucciniomycetes and the functional 
group "Plant Pathogen” were more abundant in site QJ than in site XC (Fig. S1a, b), regardless of crop species. In terms of crop species, maize possessed the highest number of specific ZOTU (Fig. S4a-c), and more abundant Glomeromycetes and "Arbuscular Mycorrhizal” group were found in maize root than in wheat and barley roots (Fig. S1a, b). LEfSe further identified some members within Pucciniaceae as important biomarker taxa for wheat and barley (Fig. S4d).

\section{The drivers and potential ecological functions of abundant and rare sub-communities}

NMDS ordinations and nested PERMANOVA analysis of the full dataset suggested that mycobiome assembly was mainly explained by compartment niche $\left(R^{2}=38.4 \%, p<0.001\right)$ and crop species $\left(R^{2}=10.5 \%, p<0.001\right)$, rather than by site $\left(R^{2}=0.9 \%, p<0.001\right)$ and fertilization practice $\left(R^{2}=0.9 \%, p=0.015\right)$ (Fig. 2a, Table S2). Importantly, fertilization practice had significant influence on abundant sub-community $\left(R^{2}=0.9 \%, p<0.001\right)$ but did not on rare sub-community $\left(R^{2}=1.0 \%, p=0.116\right)$ (Table S2). Within each compartment niche, soil mycobiomes were primarily shaped by site (explained $~ 60 \%$ variation in whole community, $p<$ 0.001), and phylloplane and rhizoplane mycobiomes were co-influenced by both host species $(\sim 49 \%, p<0.001)$ and site $(\sim 25 \%, p<0.001)$ (Fig. S5a-c). By contrast, root and leaf endosphere mycobiomes were shaped primarily by host species $(\sim 33 \%, p<0.001)$ rather than by site $(\sim 4 \%, p$ $<0.001$ ) regardless of the whole, abundant or rare communities (Fig. S5a-c). Moreover, root and leaf endosphere fungal communities had the greater beta-diversity than any other compartment niches (Fig. 2b). In addition, fungal Shannon index gradually decreased from soils ( 6.2 in bulk 
soil and rhizosphere) to epiphytes ( 4.7 in rhizoplane and phylloplane) and to endophytes ( 3.2 in root and leaf endosphere) (Fig. 2b). Strikingly, site QJ (acidic red soil) had greater Shannon index than site XC (fluvo-aquic soil) in each compartment niche except for root and leaf endosphere (Fig. S6). All these results indicated that crop hosts had the strongest selection effect on endosphere fungal communities.

Mantel test further suggested that $\mathrm{pH}$ and $\mathrm{NO}_{3}{ }^{-}-\mathrm{N}$ were the strongest environmental variables influencing fungal communities in the rhizosphere and bulk soils (Fig. 3a; Table S3). Rare sub-community had stronger correlations with soils nitrogenase activity, $\alpha$-glucosidase, and $\beta$-xylosidase, while abundant sub-community had stronger correlations with soils potential nitrification rate, denitrifying enzyme activity, and $\beta$-D-cellobiosidase (Fig. 3a; Table S3). We further performed Random Forest modelling analysis to identify the most important predictors (with higher value of Increase in MSE) for crop yield. The results showed that the community composition and functional guilds of rare taxa played a stronger role in predicting crop yield than abundant taxa did (Fig. 3b), highlighting the importance of rare taxa for crop host. Moreover, the fungal communities in rhizoplane, phylloplane and rhizosphere had more important roles in predicting crop yield than other compartments.

\section{Rare taxa are more sensitive to host selection but more stable to environmental perturbation}

Null model analyses showed that the influence of determinism (i.e. $\beta$ NTI $\geqslant 2$ or $\beta$ NTI $\leqslant$ -2) and stochasticity (i.e. $-2<\beta$ NTI $<2$ ) on mycobiome assembly varied between soil and plant 
compartment niche, with mycobiomes (including whole, abundant and rare communities) in rhizosphere and bulk soils dominated by deterministic processes ( $65 \%$ of samples were dominated by deterministic processes) (Fig. 4a). As for 4 plant compartment niches, abundant sub-community was dominated by stochastic processes ( 69\%), while rare sub-community was mainly determined by deterministic processes ( 58\%) (Fig. 4a).

Source-tracking analysis was further conducted to identify the potential sources of fungal communities in each host compartment and assess the effect of host selection on abundant and rare taxa. Our results showed that crop-associated fungal communities were derived primarily from bulk soils and gradually enriched and filtered by different compartment niches (Fig. 4b). As for the whole and abundant communities, rhizosphere mycobiomes were derived primarily from bulk soils and were the main source of rhizoplane. Successively, rhizoplane served as the main source not only for root endophytes but also for plant above-ground compartments (Fig. 4b, Fig. S7a-c). Moreover, we found higher unknown source in maize leaf epiphytes ( 46\%), compared to wheat ( 8\%) and barley ( 5\%) (Fig. S7a-c). The rare sub-community displayed similar source patterns as the whole and abundant communities, but had higher unknown sources for each compartment niche. Similar source patterns were observed for each crop species (Fig. 4b, Fig. S7a-c). The differential abundance analysis for each individual compartment niche versus bulk soil further indicated that the enriched and depleted ZOTUs were mainly grouped to rare taxa (Fig. S8a-b). The depleted index (DI) value was found to be gradually increased from rhizosphere (0.54) to 
epiphytes (1.39-1.69) and to endophytes (3.16-4.9) (Fig. S8a). Among 46 ZOTUs significantly enriched and overlapped in 4 plant compartment niches, and 21 ZOTUs were affiliated within Dothideomycetes (Fig. S8b). By contrast, 734 ZOTUs were significantly depleted in 4 plant compartment niches, and $\sim 41 \%$ of them belonged to Sordariomycetes (304 ZOTUs) (Fig. S8b). The Venn plot also showed that the majority of specific ZOTUs for different compartment niches and crops belonged to rare taxa (Fig. S4a-c). Within each plant compartment niche, the core ZOTUs (present in at least $80 \%$ of samples) were mainly grouped to abundant taxa, then to moderate taxa, but not to any rare taxa (Table S4). Notably, we found that ZOTU1 (identified as Alternaria) and ZOTU2 (Epicoccum) were the most abundant core ZOTUs in each plant compartment niche. Furthermore, the lowest ZOTU number, retrieved sequences number, and diversity index of rare sub-community were recorded in leaf endosphere (Table S1 and S4). All these results indicated that rare taxa were more sensitive to host selection mediated by both compartment niche and host species than abundant taxa.

Functional guilds prediction suggested that rare taxa were more stable than abundant taxa under different fertilization practices (Table S5). Nested PERMANOVA analysis based on full dataset showed that fertilization practice had a significant influence on fungal functional guilds in abundant sub-community $\left(R^{2}=1.5 \%, p<0.001\right)$ but did not on rare sub-community $\left(R^{2}=0.7 \%, p\right.$ $=0.580$ ) (Table S5). To exclude the bias from different crop species, we repeated analysis only on maize samples and found similar pattern as the analysis based on full dataset (Table S2, Table S5). 
The results based on maize dataset further demonstrated that fertilization practice had significant influence on the relative abundance of functional group "Plant Pathogen" in abundant sub-community, but did not on group “Arbuscular Mycorrhizal” in rare sub-community (Fig. S9, Fig. S10). $\mathrm{N}$ treatment (local $\mathrm{N}$ fertilization regime) significantly increased the relative abundance of group "Plant Pathogen" in rhizoplane in site XC, compared to $20 \% \mathrm{~N}$ reduction treatment $(80 \% \mathrm{~N})(p=0.009)$. $\mathrm{N}$ and $80 \% \mathrm{NI}(80 \% \mathrm{~N}$ treatment plus nitrification inhibitor) treatments significantly increased the relative abundance of "Plant Pathogen” in leaf endosphere in site QJ, compared with $80 \% \mathrm{~N}(p=0.002)$ (Fig. S9).

\section{Rare taxa play a key role in fungal co-occurrence network}

We further performed network analysis to disentangle the ecological role and co-occurrence patterns of abundant and rare taxa along the soil-plant continuum, and showed that rare taxa played an important role in fungal co-occurrence network (Fig. 5a-d). The network was composed of 2013 edges and 345 nodes which included 8.12\% abundant ZOTUs, 20.58\% rare ZOTUs, and 71.30\% moderate ZOTUs, and were mainly represented by Dothideomycetes (31.59\%) and Sordariomycetes (29.57\%) (Fig. 5a, c). Intriguingly, rare ZOTUs had significantly higher values of the degree and closeness centrality than both abundant and moderate ZOTUs in the network (Fig. 5d). We further defined "Hub species" as ZOTU with high values of degree (> 50) and closeness centrality (> 0.6) in the network. Thus 14 hub species composing of 11 rare ZOTUs and 3 moderate ZOTUs were identified, and these hub species were mainly affiliating within 
Dothideomycetes (Fig. 5c, d). Among these hub species, top three ZOTUs with the highest degree and closeness centrality values belonged to rare taxa and classified into Periconia, Paraconiothyrium, and Coniella, respectively.

Moreover, the network modules constituted of different taxa and exhibited distinct distribution patterns along the soil-plant continuum. The fungal network was clearly grouped into 6 major ecological clusters (modules), of which module \#1 and module \#2 together comprised more than $50 \%$ of the whole network node (Fig. 5b). The module \#1, \#3, and \#5 were mainly composed of abundant and moderate ZOTUs, and primarily represented by Sordariomycetes. By contrast, the module \#2, \#4, and \#6 were mainly composed of rare and moderate ZOTUs, and primarily represented by Dothideomycetes (Fig. 5b, c). Additionally, we found that the distribution patterns of these modules differed between soil and plant compartment niches. The relative abundance of module \#2, \#4, and \#6 were more abundant in phylloplane, leaf endosphere, and root endosphere than in rhizosphere and bulk soils $(p<0.001)$, while the module \#1 was much higher in soils than in plant compartment niches $(p<0.001)$ (Fig. 5e). Functional guilds prediction on different modules further suggested that "Dung Saprotroph" (26.2\%) was the top abundant functional group in module \#1, \#3, \#5, while “Plant Pathogen” (20.4\%) topped in module \#2, \#4, \#6. 


\section{Discussion}

\section{Dominant host selection in shaping crop fungal communities}

Uncovering the mechanisms of plant microbiome assembly and host-microbe-environment interactions is a central goal for understanding fundamental ecological processes and harnessing the plant microbiomes to maximize agriculture production (Singh \& Trivedi, 2017). In this study, a scenario survey on fungal communities along the soil-plant continuum in field-grown crops suggests that mycobiome assembly is shaped largely by host selection (i.e. compartment niche and crop species) at the plant level, rather than by site and fertilization practice. This finding is supported by previous studies showing that plant leaf, root and rhizosphere niches are colonized by distinct fungal communities (Yu et al., 2018; Gao et al., 2020). In terms of a specific niche, soil mycobiomes varied largely between two sites, with site-dependent variables like soil $\mathrm{pH}$ and nutrients as the driving factors, which were consistent with numerous studies in various soil ecosystems (Shi et al., 2018; Jiao et al., 2020). In contrast, phylloplane and rhizoplane mycobiomes were influenced by both host species and sites, and root and leaf endosphere mycobiomes were shaped primarily by host species. Similarly, some previous studies also showed that fungal communities in a specific compartment niche like phyllosphere, rhizosphere and root are mainly affected by host genetics, and co-influenced by soil characteristics, climate and geographic origin (Sapkota et al., 2015; Yu et al., 2018; Koyama et al., 2019; Yao et al., 2019). We further found that fungal Shannon index decreased while community dissimilarity and depleted 
index (DI) increased from soils to epiphytes and to endophytes. Collectively, these findings suggest that host selection plays a predominant role in shaping crop mycobiomes, with the strongest host selection effect on crop endophytes. Similar observations were also recorded in microbial communities of Arabidopsis roots (Duran et al., 2018) and bacterial microbiome in our previous study (Xiong et al., 2020). This could be the consequence of intensive selection pressures caused by host immune system in endophytic niches (Guttman et al., 2014; Hacquard et al., 2015). Our study further advances the discipline by providing systematic and empirical evidence for the theoretical host selection and niche occupation theory for mycobiome assemblies across rhizosphere, phyllosphere and endosphere in the maize-wheat/barley rotation system under different environmental conditions.

The source-tracking analysis reveals that rhizosphere mycobiomes are primarily derived from bulk soils, and gradually filtered and enriched by rhizoplane and other plant compartments, indicating that soil habitats are the major sources for crop microbial selection. We found higher unknown source in maize leaf epiphytes compared to wheat/barley, which may be the consequence of the difference in plant functional traits (e.g. leaf area) and season-dependent factors (e.g. precipitation) between maize and wheat/barley (Vandenkoornhuyse et al., 2015; Muller et al., 2016). Moreover, abundant taxa were highly widespread while rare taxa were habitat-specific and more sensitive to host selection than abundant taxa. Further, we found that the deterministic processes (i.e. niche theory) dominated over the stochastic processes (i.e. neutral 
theory) in rare sub-community while it was on reverse for the abundant sub-community in 4 plant compartment niches, further confirming the pronounced host selection effect on rare taxa. These results were consistent with a previous study on bacterioplankton showing that rare taxa in lakes and reservoirs across large geographic distance were influenced primarily by local environmental variables (i.e. deterministic processes) (Liu et al., 2015). Nevertheless, it was also found that the stochastic processes played a more important role for rare taxa in a reservoir after cyanobacterial blooming and oil-contaminated soils (Jiao et al., 2017; Xue et al., 2018). This inconsistency could be explained as that the relative effects of deterministic and stochastic processes on rare sub-community are dependent on geographic scales and strength of environmental gradients (Hanson et al., 2012; Morrison-Whittle \& Goddard, 2015). Together these results support the eco-evolutionary theory that abundant taxa have the capability of utilizing a broad range of resources and overcome biotic and abiotic stress, and thus are able to adapt to different hosts and habitats through active growth and high competitive ability. By contrast, rare taxa have narrow niche breadth, less competitive ability and low growth rate, thereby their distributions are restricted by the host selection and driven largely by deterministic processes (Liu et al., 2015; Lynch \& Neufeld, 2015; Jousset et al., 2017).

\section{The important ecological role of rare taxa}

Disentangling the ecological role and co-occurrence network of abundant and rare taxa along the soil-plant continuum could broaden our understanding on species coexistence 
mechanisms (Agler et al., 2016; van der Heijden \& Hartmann, 2016). In the present study, we provide field evidence showing that mycobiomes across soil, rhizosphere, phyllosphere and endosphere are dominated by few abundant taxa, while the diversity is dominantly represented by highly diverse rare taxa. Previous studies on soil and freshwater ecosystems have reported similar observations for abundant and rare taxa (Jiao et al., 2017; Xue et al., 2018; Egidi et al., 2019), indicating such distribution is common across different habitats. Furthermore, we found that most hub species of fungal co-occurrence network belonged to rare taxa. The hub species have been proposed as "ecosystem engineers" owing to their critical role in the community assembly (Agler et al., 2016; van der Heijden \& Hartmann, 2016; Banerjee et al., 2018). Along with a similar finding in eukaryotic plankton (Xue et al., 2018), our results suggest that rare taxa play a key role in maintaining the stability of crop fungal network.

We showed that fungal network module \#1 was more abundant in soils than in plant compartment niches, and mainly composed of abundant and moderate ZOTUs affiliating within Sordariomycetes. By contrast, module \#2, \#4, and \#6 were more abundant in plant compartment niches than in soils, and mainly composed of rare and moderate ZOTUs affiliating within Dothideomycetes. Sordariomycetes is one of the largest classes of Ascomycota and widely distributed in soil, freshwater and marine habitats (Maharachchikumbura et al., 2016; Egidi et al., 2019), while Dothideomycetes was supposed to be composed of a highly diverse range of fungi, including epiphytes, endophytes, and important plant pathogens (Hyde et al., 2013). These 
together our results suggest that the clustering modules in fungal network compose of different taxa and exhibit different ecological functions, while each network module may be composed of diverse microbial groups with similar habitats adaptation and similar ecological functions (Delgado-Baquerizo et al., 2018). Our findings on the equivalent contribution of rare taxa in plant-associated ecological modules further support that rare taxa play an essential role in plant mycobiomes.

Further, our results suggest that the community composition and functional guilds of rare taxa are more stable than abundant taxa under different fertilization practices. These observations support the hypothesis that rare taxa may contribute to the resilience of the microbial community as a seed bank, and also to the sustaining of microbiome function under stress as they are supposed to be highly resistant to stress and functionally overlapped (Jousset et al., 2017; Ziegler et al., 2018). In contrast, environmental disturbances may have stronger effects on abundant taxa, as they may keep the large population size and perform the main functions through active growth (Pedros-Alio, 2012; Xue et al., 2018). Similarly, previous works have shown that rare symbionts may play an important role in host resilience due to its high function redundancy (Mouillot et al., 2013; Ziegler et al., 2018). Moreover, we found that rare taxa accounted for greater contributions to soil C and N cycling functions (soil enzymes) and crop yield prediction than abundant taxa did, and that the class Glomeromycetes was exclusively present in rare sub-community. Similarly, previous works have indicated that soil rare taxa play a more 
important role in driving soil multifunctionality than abundant taxa (Chen et al., 2020), and soil rare microbes could contribute to plant protection and productivity (Hol et al., 2010; Hol et al., 2015). Our findings corroborated these observations further suggested that rare taxa are greatly associated with microbiome stability, ecosystem functioning and plant performance (Van der Heijden et al., 1998; Van Der Heijden et al., 2015).

\section{Changes in composition and functional guilds of mycobiomes under different management}

\section{conditions}

Fungal communities along the soil-plant continuums were dominated by Ascomycota which is also a dominant soil fungal group globally (Egidi et al., 2019). Fungal community composition at the class level largely varied among different compartment niches, with Dothideomycetes and Sordariomycetes significantly enriched in plant compartment niches and soils, respectively. Dothideomycetes comprise a highly diverse range of fungi such as endophytes, epiphytes, fungicolous and plant pathogens (Hyde et al., 2013), which well explained enrichment of Dothideomycetes in plant compartment niches in our study. Intriguingly, we found that ZOTU1 (Alternaria) and ZOTU2 (Epicoccum) (both belonging to Dothideomycetes) were the most abundant core ZOTUs in all 4 plant compartment niches. Alternaria is well known as a wind-dispersed fungal genus, which comprises some important plant pathogens species (Egidi et al., 2019). In contrast, previous study has shown that Epicoccum are important endophytic fungi, which is associated with production of secondary metabolites, and that some species like $E$. 
nigrum could be able to promote plant growth and resist the brown rot pathogen as biocontrol agent (Larena et al., 2005; Lima et al., 2012).

We further showed that the class Pucciniomycetes was significantly more abundant in leaf endosphere than in other compartment niches, and that the relative abundance of both Pucciniomycetes and group "Plant Pathogen” were much higher in site QJ than in site XC. Pucciniomycetes are well-known plant parasite fungi (Henk \& Vilgalys, 2007). QJ site is located in southwest China with a subtropical monsoon climate $(1,008 \mathrm{~mm}$ annual mean precipitation, amp) and characterized with acidic red soil (with $\mathrm{pH}$ at about 5.0), while XC site was located in north China characterized with a temperate monsoonal climate (704 mm amp) and alkaline soils with $\mathrm{pH}$ at about 7.5. The relatively wet weather and low soil $\mathrm{pH}$ is known to favor the growth of Pucciniomycetes fungi (Alexander, 1980). Moreover, we found significant changes in "Plant Pathogen” group but did not in "Arbuscular Mycorrhizal” group under different fertilization practices. High chemical $\mathrm{N}$ fertilizer dosage ( $\mathrm{N}$ treatment) increased the relative abundance of potential plant pathogen in leaf endosphere and rhizoplane, consistent with previous studies (Huber \& Watson, 1974; Dordas, 2008). These results, therefore, suggest that excessive chemical $\mathrm{N}$ fertilizer input may increase the risk of plant diseases, highlighting the importance of sustainable fertilization practice.

Furthermore, we found that all members within the class Glomeromycetes (AMF) belonging to rare taxa and were significantly more abundant in root endosphere than in other compartment 
niches, and in maize roots than in wheat/barley roots. AMF form mutualistic associations with roots of the majority of terrestrial plant species and contributes significantly to plant nutrient uptake and global phosphate cycling (Van der Heijden et al., 1998; Parniske, 2008). Our findings further confirm that AM fungi associations are mainly based on root endosphere niche and maize species are better colonized by AMF compared to wheat/barley species (Fuchs \& Haselwandter, 2004; Parniske, 2008). In contrast, some members within Pucciniaceae were identified as important biomarker taxa for barley and wheat. All these results support that crop host and specialized fungal taxa mutually select each other and co-evolve in long-term scale (Guttman et al., 2014).

\section{Conclusions}

In this study, we provide a holistic understanding on the ecological processes that underpin the assembly of plant mycobiomes, and demonstrate that rare taxa play a central role in the crop fungal network and ecosystem functioning. Our results provide strong field evidences showing that mycobiome assembly along the soil-plant continuum is shaped largely by host selection (i.e. compartment niche and crop species) rather than by environmental factors. Within each plant compartment niche, mycobiomes are mainly driven by crop species, with the strongest host effect on endophytes. Moreover, rare fungal taxa are habitat-specific and represent the highly diverse fungal seed bank (e.g. AMF), whereas abundant taxa are widespread and dominate mycobiomes. 
Fungal rare taxa are more greatly affected by host selection but not by environmental perturbation (e.g. field fertilization practice), and are primarily determined by the deterministic processes in plant compartment niches, in comparison to abundant taxa. These findings hint us that future microbial ecology studies may need to differentiate rare and abundant sub-communities.

Furthermore, we demonstrate that rare taxa play a central role in fungal co-occurrence network and are greatly associated with crop yield and soil C, N cycling function like soils $\alpha$-glucosidase and nitrogenase activity. We also provide new evidence of gradual filtration of fungal communities from bulk soils to different plant compartment niches, with more abundant Sordariomycetes in soils and more Dothideomycetes in plants. These results significantly advance our fundamental understanding on ecological processes of soil-plant mycobiome assembly and highlight the ecological importance of rare taxa, which can be harnessed in the future for sustainable agriculture production.

\section{Materials and methods}

\section{Experimental treatments and sampling}

Two crop field trials were located in Xuchang, Henan province (XC, 34 $08^{\prime} 20.4^{\prime \prime} \mathrm{N}$, $113^{\circ} 48$ '34.9"E, northern China) with a neutral $\mathrm{pH}$ at about 7.5, and Qujing, Yunnan province (QJ, $25^{\circ} 09^{\prime} 40.8^{\prime \prime} \mathrm{N}, 104^{\circ} 01^{\prime} 51.5^{\prime \prime E}$, southwest China) with low pH at about 5.0. Soils at XC and QJ were classified as calcaric cambisols and chromic cambisols according to the FAO soil 
classification system (IUSS Working Group WRB, 2007). The two study sites are about $1800 \mathrm{~km}$ far away, and had totally different climate, soil parent material conditions and farming habits (Xiong et al., 2020). Both field trials were established in spring 2016 and included seven fertilization treatments: (1) Control (zero N); (2) N (local farmers' $\mathrm{N}$ rate at $200 \mathrm{~kg} \mathrm{ha}^{-1}$ season $^{-1}$ ); (3) $80 \% \mathrm{~N}(20 \% \mathrm{~N}$ reduction on the basis of $\mathrm{N}$ treatment); (4) $80 \% \mathrm{NS}$ ( $80 \% \mathrm{~N}$ treatment plus straw covering at a rate of $3000 \mathrm{~kg} \mathrm{ha}^{-1}$ season $\left.^{-1}\right)$; (5) $80 \% \mathrm{NI}(80 \% \mathrm{~N}$ treatment plus nitrification inhibitor chlorinated pyridine at a rate of $\left.735 \mathrm{~g} \mathrm{ha}^{-1}\right)$; (6) $80 \% \mathrm{NKle}(80 \% \mathrm{~N}$ treatment plus foliar spraying of an asymbiotic nitrogen-fixing bacteria Klebsiella variicola W12 at a rate of $500 \mathrm{~L} \mathrm{ha}^{-1}$, $1 \times 10^{12} \mathrm{CFU} / \mathrm{ml}$ ); (7) $80 \% \mathrm{NSB}$ (80\%NS treatment plus biochar addition at a rate of 30,000 kg ha ${ }^{-1}$ every two years). Each fertilization treatment had three replicate plots (about $30 \mathrm{~m}^{2}$ ), and all plots were randomly arranged in field and managed according to local habits. The crop varieties planted were the same as those used by local farmers, with maize cultivar Zhengdan 958 and wheat cultivar Qiule 2122 sowed in XC, and maize cultivar Shidan 8 and barley cultivar V43 in QJ.

Sampling was performed at grain filling stage of wheat or barley in April 2017 (the first 6 treatments), and tasseling stages of maize in August 2017 (all 7 treatments) for both sites. For each replicate plot, about 5 individual maize and 20-30 individual wheat/barley were sampled for leaves and roots samples. For each individual plant, 1-2 leaves and roots at equivalent position were collected and totally 50-100 g leaf and 10-20 g root (fresh weight) samples were obtained for 
each plot. Rhizosphere soil was defined as the soil particles tightly attached to roots, and was collected by shaking the roots strongly. The bulk soils were collected at $\sim 20 \mathrm{~cm}$ away from axial roots with a depth of 0-15 cm, and five subsamples from the same plants used for rhizosphere soil collection in each plot were mixed as a biological replicate. All samples for molecular works were transported to the laboratory on dry ice and stored at $-80{ }^{\circ} \mathrm{C}$ until further processing. The crop yield was measured based on the field harvest according to previous study (Zhang et al., 2016). Soil physicochemical parameters (including $\mathrm{pH}, \mathrm{NH}_{4}{ }^{+}-\mathrm{N}, \mathrm{NO}_{3}{ }^{-}-\mathrm{N}$, dissolved organic carbon, and dissolved organic nitrogen) and enzyme activities related with $\mathrm{C}, \mathrm{N}$, and $\mathrm{P}$ cycling (e.g. nitrogenase activity and phosphatase) were measured according to standard protocols (Tabatabai, 1994; Marx et al., 2001; Zhao et al., 2019). More detailed descriptions on field management and the methods for soil properties measurement are available in Supplementary Information (Method S1).

\section{DNA extraction and fungal community amplicon sequencing}

For epiphytic DNA extraction, 10-15 g of healthy leaves or 3-5 g roots were put in sterile bottle or polystyrene tubes containing release buffer and microbial cells were collected according to our previous study (Xiong et al., 2020), and subjected to DNA extraction using the PowerSoil DNA Isolation Kit (MO BIO Laboratories, Carlsbad, CA, USA). Endophytic microbial DNA was extracted from the same leaves and roots used for epiphytic DNA after further surface sterilization (Ruiz-Perez et al., 2016; Xiong et al., 2020). The rhizosphere and bulk soil DNA were 
extracted from $0.4 \mathrm{~g}$ soil using the PowerSoil DNA Isolation Kit. Totally, 468 DNA samples covering 6 compartment niches were obtained (wheat/barley: $n=6$ treatments $\times 3$ replicates $\times 2$ sites $\times 6$ compartments $=216$; maize: $\mathrm{n}=7$ treatments $\times 3$ replicates $\times 2$ sites $\times 6$ compartments $=252$ ), and subjected to fungal ITS2 region amplification using primers fITS7 (Ihrmark et al., 2012) and ITS4 (White et al., 1990). The PCR products were cleaned using the QIAquick gel extraction kit (Qiagen, USA) and samples were combined in equimolar concentrations into one library. Libraries were sequenced on the Illumina MiSeq platform with a Paired-End protocol. More detailed descriptions on DNA extraction and fungal ITS2 region amplification are available in Supplementary Information (Method S1).

\section{Sequence processing}

Amplicon data were analysed using a combination of the USEARCH v10 (Edgar, 2010) and QIIME v1.91 (Caporaso et al., 2010) software. Briefly, primer sequences and low-quality reads ends with a quality score (Q) below 30 were trimmed. Raw forward and reverse reads for each sample were merged to a single sequence, and the paired reads were trimmed to $200 \mathrm{bp}$ and then quality-filtered (maximum expected error 0.5) in USEARCH. All qualified biological reads (called zero-radius OTUs, or ZOTUs) were picked at $100 \%$ sequence similarity using unoise3 command (Edgar, 2016) with default parameters in USEARCH. Taxonomy was assigned using BLAST algorithm with the UNITE database (v7.0) in QIIME. Fungal ZOTUs assigned to plant or protist were removed, and ZOTUs represented by less than 2 sequences were removed to avoid 
possible biases. A total of 22,598,848 high quality sequences from 468 samples were obtained with a mean of 48,288 $\pm 18,895$ reads per sample, and these reads were sorted into 7,699 zero-radius operational taxonomic units (ZOTUs). The ZOTU table was rarefied to 4720 reads for alpha diversity estimates among different compartment niches, and MetagenomeSeq's cumulative sum scaling (CSS) was used as a normalization method for beta-diversity analyses. Both alpha-diversity and beta-diversity of fungal community were calculated in QIIME.

We defined abundant or rare ZOTUs following previously suggested rules that combined local and regional relative abundances of microbial taxa (Liu et al., 2015). Briefly, abundant ZOTUs were defined as the ZOTUs with local relative abundances $\geq 1 \%$ within a sample and regional relative abundances $\geq 0.1 \%$ across all samples, whereas rare ZOTUs were defined as the ZOTUs with local relative abundances $<0.01 \%$ within a sample and regional relative abundances $<0.001 \%$ across all samples. Those ZOTUs neither belonging to abundant taxa nor rare taxa were defined as moderate ZOTUs.

\section{Statistical analysis}

To explore the species trait and ecological adaptation of abundant and rare fungal taxa, we calculated Levin's niche breadth index separately for the abundant and rare taxa using "spaa” $\mathrm{R}$ package (Zhang, 2004). We further explored the relationship between niche breadth and relative abundance of different fungal taxa by correlation analysis and quadratic regression analyses. The phylogenetic tree was constructed using FastTree (based on the Maximum Likelihood method) in 
QIIME, and annotated and visualized in iTOL software (Letunic \& Bork, 2019). The fungal functional guilds were predicted using the program FUNGuild (Nguyen et al., 2016). Linear discriminant analysis (LEfSe) was applied (Wilcoxon p-value: 0.05, LDA > 2; http://huttenhower.sph.harvard.edu/galaxy/) to identify the biomarker of each compartment niche and crop. Weighted UniFrac distances between samples were calculated and visualized using non-metric multi-dimensional scaling (NMDS) ordinations to assess fungal beta-diversity. The significance of different factors on fungal community and functional guilds was tested with PERMANOVA or nested PERMANOVA (crop species nested within fertilization treatment) using the "adonis" function of the "vegan” package (Oksanen et al., 2007). The correlations between fungal communities (based on Bray-Curtis community dissimilarity), soil physicochemical characteristics, and soil enzyme activities in rhizosphere and bulk soil were assessed by Mantel tests. Random forest modeling using the "randomForest” R package (Liaw \& Wiener, 2002) were conducted to identify the most important predictor of crop yield (Xiong et al., 2020). The significance of the model and each predictor was assessed using the "A3" and "rfPermute" $\mathrm{R}$ package, respectively (Fortmannroe, 2015; Delgado-Baquerizo et al., 2016).

To determine the potential contribution of deterministic and stochastic processes on microbiome assembly, we calculated the beta Nearest Taxon Index ( $\beta$ NTI) values using null model (999 randomizations) (Stegen et al., 2013) in R, and $|\beta N T I| \geq 2$ and $|\beta N T I|<2$ represent dominant deterministic processes and stochastic processes in shaping microbial community (Zhou et al., 
2014; Jiao et al., 2020), respectively. SourceTracker (v1.0) based on Bayesian approach was used to estimate the sources of the fungal communities in each plant compartment niche (Knights et al., 2011). Differentially abundance analysis was performed using EdgeR's generalized linear model (GLM) approach (Robinson et al., 2010). The "Depleted index" (DI) and "Dissimilarity index" (DSI) were defined to assess fungal selection processes from bulk soil to other compartment niches. The network based on Spearman correlation scores were calculated using the CoNet (Faust et al., 2012) in Cytoscape v3.5 (Shannon et al., 2003), and only robust (Spearman's $r>0.8$ or $r<$ -0.8) and statistically significant $(p<0.01)$ correlations were kept. The "benjaminihochberg" multiple testing correction $(p<0.05)$ was performed before network construction. The ZOTUs with low frequency (present in $<20$ samples) were removed from network analysis to avoid possible biases, as a large number of zeros values can introduce spurious correlations (Xue et al., 2018). Finally, a total of 3808 ZOTUs were used for the network analyses. The network was visualized in Gephi (Bastian et al., 2009) and Cytoscape. The topology characteristics (e.g. degree, betweenness centrality, and closeness centrality) of the network were calculated using Network Analyzer plugin in Cytoscape. Network modular analysis was conducted in Gephi with default parameters, and the relative abundance of each module was computed by averaging the standardized relative abundances (z-score) of the taxa that belong to each module (Delgado-Baquerizo et al., 2018). Fungal ZOTU with high degree (> 50) and closeness centrality values (> 0.6) were identified as "hub species" in co-occurrence networks according to 
previous studies (Agler et al., 2016; van der Heijden \& Hartmann, 2016).

All statistical analyses were carried out in R (http://www.r-project.org). Nonparametric statistical tests have been run to evaluate the community attributes (e.g. the significance of difference in taxonomic composition, alpha-diversity, and functional group) observed among 6 different compartment niches (Kruskal-Wallis test or Wilcoxon test, $p<0.05$ ). For multiple comparison tests, a false discovery rate (FDR) has been applied. More information on the definition of DSI and DI, and the methods of Random forest modeling analysis and source tracking analysis are detailed in our previous study (Xiong et al., 2020) and the Supplementary Material (Method S1).

\section{Acknowledgements}

We would like to thank Prof. Yan-Lai Han, Mr. Li-Hua Wan and Hao-Tian Tong for their cooperation and assistance in the field work, and Zi-Yang He, Zhi-Bo Zhao, and Chang-Chun Zhai for their generous helps in soil sampling and laboratory work. We also thank Dr. Hang-Wei Hu for his assistance in data analysis. This work was financially supported by National Key R\&D Program (2017YFD0200600) and the Strategic Priority Research Program (B) of Chinese Academy of Sciences (XDB15020200) and Research on plant and soil microbiomes in BKS lab is supported by Australian Research Council (DP170103628; DP190103714). Li-Mei Zhang was supported by the Youth Innovation Promotion Association, Chinese Academy of Sciences. 


\section{Data availability}

All raw sequencing data have been submitted to the NCBI Sequence Read Archive (SRA)

database under the accession number PRJNA627136.

\section{Conflict of Interest}

The authors declare that they have no conflict of interest. 


\section{References}

Agler M.T., Ruhe J., Kroll S., Morhenn C., Kim S.T., Weigel D., Kemen E.M. (2016) Microbial hub taxa link host and abiotic factors to plant microbiome variation. PLoS Biology 14: e1002352.

Alexander M. (1980) Effects of acid precipitation on terrestrial ecosystems. In Effects of acidity on microorganisms and microbial processes in soil. Hutchinson T.C., Havas M. (eds). Boston: Springer US, pp. 363-374.

Almario J., Jeena G., Wunder J., Langen G., Zuccaro A., Coupland G., Bucher M. (2017) Root-associated fungal microbiota of nonmycorrhizal Arabis alpina and its contribution to plant phosphorus nutrition. Proc. Natl. Acad. Sci. U S A 114: E9403-E9412.

Banerjee S., Schlaeppi K., van der Heijden M.G.A. (2018) Keystone taxa as drivers of microbiome structure and functioning. Nat. Rev. Microbiol. 16: 567-576.

Bastian M., Heymann S., Jacomy M. (2009) Gephi: an open source software for exploring and manipulating networks. Icwsm 8: 361-362.

Boulotte N.M., Dalton S.J., Carroll A.G., Harrison P.L., Putnam H.M., Peplow L.M., van Oppen M.J. (2016) Exploring the Symbiodinium rare biosphere provides evidence for symbiont switching in reef-building corals. ISME J 10: 2693-2701.

Bulgarelli D., Garrido-Oter R., Munch P.C., Weiman A., Droge J., Pan Y. et al. (2015) Structure and function of the bacterial root microbiota in wild and domesticated barley. Cell Host Microbe 17: $392-403$.

Bulgarelli D., Schlaeppi K., Spaepen S., Ver Loren van Themaat E., Schulze-Lefert P. (2013) Structure and functions of the bacterial microbiota of plants. Annual Review of Plant Biology 64: 807-838.

Caporaso J., Kuczynski J., Stombaugh J., et al. (2010) QIIME allows analysis of highthroughput community sequencing data. Nat. Methods 7: 335-336.

Chen Q.L., Ding J., Zhu D., Hu H.W., Delgado-Baquerizo M., Ma Y.B. et al. (2020) Rare microbial taxa as the major drivers of ecosystem multifunctionality in long-term fertilized soils. Soil Biology and 
Biochemistry 141: 107686.

Christian N., Herre E.A., Clay K. (2019) Foliar endophytic fungi alter patterns of nitrogen uptake and distribution in Theobroma cacao. New Phytologist 222: 1573-1583.

Cregger M.A., Veach A.M., Yang Z.K., Crouch M.J., Vilgalys R., Tuskan G.A., Schadt C.W. (2018) The Populus holobiont: dissecting the effects of plant niches and genotype on the microbiome.

Microbiome 6: 31.

Delgado-Baquerizo M., Maestre F.T., Reich P.B., Jeffries T.C., Gaitan J.J., Encinar D. et al. (2016) Microbial diversity drives multifunctionality in terrestrial ecosystems. Nat. Commun. 7: 10541.

Delgado-Baquerizo M., Reith F., Dennis P.G., Hamonts K., Powell J.R., Young A. et al. (2018)

Ecological drivers of soil microbial diversity and soil biological networks in the Southern Hemisphere. Ecology 99: 583-596.

Dordas C. (2008) Role of nutrients in controlling plant diseases in sustainable agriculture. A review. Agronomy for Sustainable Development 28: 33-46.

Duran P., Thiergart T., Garrido-Oter R., Agler M., Kemen E., Schulze-Lefert P., Hacquard S. (2018) Microbial interkingdom interactions in roots promote Arabidopsis survival. Cell 175: 973-983.e914.

Edgar R.C. (2010) Search and clustering orders of magnitude faster than BLAST. Bioinformatics 26: $2460-2461$

Edgar R.C. (2016) UNOISE2: improved error-correction for Illumina $16 S$ and ITS amplicon sequencing. BioRxiv 081257. doi: 10.1101/081257

Edwards J., Johnson C., Santos-Medellín C., Lurie E., Podishetty N.K., Bhatnagar S. et al. (2015) Structure, variation, and assembly of the root-associated microbiomes of rice. Proc. Natl. Acad. Sci. U S A 112: E911-E920.

Edwards J.D., Pittelkow C.M., Kent A.D., Yang W.H. (2018) Dynamic biochar effects on soil nitrous oxide emissions and underlying microbial processes during the maize growing season. Soil Biology and Biochemistry 122: 81-90. 
Egidi E., Delgado-Baquerizo M., Plett J.M., Wang J., Eldridge D.J., Bardgett R.D. et al. (2019) A few Ascomycota taxa dominate soil fungal communities worldwide. Nat. Commun. 10: 1-9.

Faust K., Sathirapongsasuti J.F., Izard J., Segata N., Gevers D., Raes J., Huttenhower C. (2012) Microbial co-occurrence relationships in the human microbiome. PLoS Computational Biology 8: e1002606.

Fortmannroe S. (2015) A3: accurate, adaptable, and accessible error metrics for predictive models. Journal of Statistical Software 66: 1-23.

Foster K.R., Schluter J., Coyte K.Z., Rakoff-Nahoum S. (2017) The evolution of the host microbiome as an ecosystem on a leash. Nature 548: 43-51.

Fuchs B., Haselwandter K. (2004) Red list plants: colonization by arbuscular mycorrhizal fungi and dark septate endophytes. Mycorrhiza 14: 277-281.

Gao C., Montoya L., Xu L., Madera M., Hollingsworth J., Purdom E. et al. (2020) Fungal community assembly in drought-stressed sorghum shows stochasticity, selection, and universal ecological dynamics. Nat. Commun. 11: 1-14.

Guttman D.S., McHardy A.C., Schulze-Lefert P. (2014) Microbial genome-enabled insights into plant-microorganism interactions. Nature Reviews Genetics 15: 797-813.

Hacquard S., Garrido-Oter R., Gonzalez A., Spaepen S., Ackermann G., Lebeis S. et al. (2015) Microbiota and host nutrition across plant and animal kingdoms. Cell Host Microbe 17: 603-616.

Hamonts K., Trivedi P., Garg A., Janitz C., Grinyer J., Holford P. et al. (2017) Field study reveals core plant microbiota and relative importance of their drivers. Environ. Microbiol. 20: 124-140.

Hanson C.A., Fuhrman J.A., Horner-Devine M.C., Martiny J.B.H. (2012) Beyond biogeographic patterns: processes shaping the microbial landscape. Nat. Rev. Microbiol. 10: 497-506.

Henk D.A., Vilgalys R. (2007) Molecular phylogeny suggests a single origin of insect symbiosis in the Pucciniomycetes with support for some relationships within the genus Septobasidium. American Journal of Botany 94: 1515-1526. 
Hol W.H., de Boer W., de Hollander M., Kuramae E.E., Meisner A., van der Putten W.H. (2015) Context dependency and saturating effects of loss of rare soil microbes on plant productivity. Frontiers in Plant Science 6: 485.

Hol W.H., de Boer W., Termorshuizen A.J., Meyer K.M., Schneider J.H., van Dam N.M. et al. (2010) Reduction of rare soil microbes modifies plant-herbivore interactions. Ecology Letters 13: 292-301.

Huber D., Watson R. (1974) Nitrogen form and plant disease. Annual Review of Phytopathology 12: 139-165.

Hyde K.D., Jones E.G., Liu J.K., Ariyawansa H., Boehm E., Boonmee S. et al. (2013) Families of Dothideomycetes. Fungal Diversity 63: 1-313.

Ihrmark K., Bodeker I.T., Cruz-Martinez K., Friberg H., Kubartova A., Schenck J. et al. (2012) New primers to amplify the fungal ITS2 region-evaluation by 454-sequencing of artificial and natural communities. FEMS Microbiology Ecology 82: 666-677.

Jiao S., Chen W., Wei G. (2017) Biogeography and ecological diversity patterns of rare and abundant bacteria in oil-contaminated soils. Molecular Ecology 26: 5305-5317.

Jiao S., Lu Y. (2020) Abundant fungi adapt to broader environmental gradients than rare fungi in agricultural fields. Global Change Biology 26: 4506-4520.

Jiao S., Yang Y., Xu Y., Zhang J., Lu Y. (2020) Balance between community assembly processes mediates species coexistence in agricultural soil microbiomes across eastern China. ISME J 14: 202-216.

Jousset A., Bienhold C., Chatzinotas A., Gallien L., Gobet A., Kurm V. et al. (2017) Where less may be more: how the rare biosphere pulls ecosystems strings. ISME J 11: 853-862.

Knights D., Kuczynski J., Charlson E.S., Zaneveld J., Mozer M.C., Collman R.G. et al. (2011) Bayesian community-wide culture-independent microbial source tracking. Nat. Methods 8: 761-763.

Koyama A., Maherali H., Antunes P.M. (2019) Plant geographic origin and phylogeny as potential drivers of community structure in root-inhabiting fungi. Journal of Ecology 107: 1720-1736. 
Larena I., Torres R., De Cal A., Liñán M., Melgarejo P., Domenichini P. et al. (2005) Biological control of postharvest brown rot (Monilinia spp.) of peaches by field applications of Epicoccum nigrum. Biological Control 32: 305-310.

Lau J.A., Lennon J.T. (2011) Evolutionary ecology of plant-microbe interactions: soil microbial structure alters selection on plant traits. New Phytologist 192: 215-224.

Letunic I., Bork P. (2019) Interactive Tree Of Life (iTOL) v4: recent updates and new developments. Nucleic Acids Research 47: W256-W259.

Liaw A., Wiener M. (2002) Classification and regression by randomForest. R news 2: 18-22.

Lima F.L.C.D., Souza S.F.L.D., Luiz A.W., Liles M.R. (2012) Epicoccum nigrum P16, a sugarcane endophyte, produces antifungal compounds and induces root growth. PloS One 7: e36826.

Liu L., Yang J., Yu Z., Wilkinson D.M. (2015) The biogeography of abundant and rare bacterioplankton in the lakes and reservoirs of China. ISME J 9: 2068-2077.

Lynch M.D., Neufeld J.D. (2015) Ecology and exploration of the rare biosphere. Nat. Rev. Microbiol. 13: 217-229.

Maharachchikumbura S.S., Hyde K.D., Jones E.G., McKenzie E., Bhat J.D., Dayarathne M.C. et al. (2016) Families of Sordariomycetes. Fungal Diversity 79: 1-317.

Martin F.M., Uroz S., Barker D.G. (2017) Ancestral alliances: plant mutualistic symbioses with fungi and bacteria. Science 356: eaad4501.

Marx M.C., Wood M., Jarvis S. (2001) A microplate fluorimetric assay for the study of enzyme diversity in soils. Soil Biology and Biochemistry 33: 1633-1640.

Mommer L., Cotton T.E.A., Raaijmakers J.M., Termorshuizen A.J., van Ruijven J., Hendriks M. et al. (2018) Lost in diversity: the interactions between soil-borne fungi, biodiversity and plant productivity. New Phytologist 218: 542-553.

Morcillo R.J., Singh S.K., He D., An G., Vilchez J.I., Tang K. et al. (2020) Rhizobacterium-derived diacetyl modulates plant immunity in a phosphate-dependent manner. EMBO J 39: e102602. 
Morrison-Whittle P., Goddard M.R. (2015) Quantifying the relative roles of selective and neutral processes in defining eukaryotic microbial communities. ISME J 9: 2003-2011.

Mouillot D., Bellwood D.R., Baraloto C., Chave J., Galzin R., Harmelin-Vivien M. et al. (2013) Rare species support vulnerable functions in high-diversity ecosystems. PLoS Biology 11: e1001569.

Muller D.B., Vogel C., Bai Y., Vorholt J.A. (2016) The plant microbiota: systems-level insights and perspectives. Annual Review of Genetics 50: 211-234.

Nguyen N.H., Song Z., Bates S.T., Branco S., Tedersoo L., Menke J. et al. (2016) FUNGuild: an open annotation tool for parsing fungal community datasets by ecological guild. Fungal Ecology 20:

241-248.

Oksanen J., Kindt R., Legendre P., O’Hara B., Stevens M.H.H., Oksanen M.J., Suggests M. (2007) The vegan package. Community Ecology Package 10: 631-637.

Parniske M. (2008) Arbuscular mycorrhiza: the mother of plant root endosymbioses. Nat. Rev. Microbiol. 6: $763-775$.

Pedros-Alio C. (2012) The rare bacterial biosphere. Annual Review of Marine Science 4: 449-466.

Pent M., Hiltunen M., Põldmaa K., Furneaux B., Hildebrand F., Johannesson H. et al. (2018) Host genetic variation strongly influences the microbiome structure and function in fungal fruiting-bodies. Environ. Microbiol. 20: 1641-1650.

Robinson M.D., McCarthy D.J., Smyth G.K. (2010) edgeR: a Bioconductor package for differential expression analysis of digital gene expression data. Bioinformatics 26: 139-140.

Ruiz-Perez C.A., Restrepo S., Zambrano M.M. (2016) Microbial and functional diversity within the phyllosphere of espeletia species in an andean high-mountain ecosystem. Appl. Environ. Microbiol. 82: 1807-1817.

Sapkota R., Knorr K., Jorgensen L.N., O'Hanlon K.A., Nicolaisen M. (2015) Host genotype is an important determinant of the cereal phyllosphere mycobiome. New Phytologist 207: 1134-1144.

Semchenko M., Leff J.W., Lozano Y.M., Saar S., Davison J., Wilkinson A. et al. (2018) Fungal diversity 
regulates plant-soil feedbacks in temperate grassland. Science Advances 4: eaau4578.

Shannon P., Markiel A., Ozier O., Baliga N.S., Wang J.T., Ramage D. et al. (2003) Cytoscape: a software environment for integrated models of biomolecular interaction networks. Genome Research 13: 2498-2504.

Shi Y., Li Y., Xiang X., Sun R., Yang T., He D. et al. (2018) Spatial scale affects the relative role of stochasticity versus determinism in soil bacterial communities in wheat fields across the North China Plain. Microbiome 6: 27.

Singh B.K., Liu H.W., Trivedi P. (2020) Eco-holobiont: A new concept to identify drivers of host-associated microorganisms. Environ. Microbiol. 22: 564-567.

Singh B.K., Trivedi P. (2017) Microbiome and the future for food and nutrient security. Microbial Biotechnology 10: 50-53.

Stegen J.C., Lin X., Fredrickson J.K., Chen X., Kennedy D.W., Murray C.J. et al. (2013) Quantifying community assembly processes and identifying features that impose them. ISME J 7: 2069-2079.

Sun R.B., Zhang X.X., Guo X.S., Wang D.Z., Chu H.Y. (2015) Bacterial diversity in soils subjected to long-term chemical fertilization can be more stably maintained with the addition of livestock manure than wheat straw. Soil Biology and Biochemistry 88: 9-18.

Tabatabai M. (1994) Methods of Soil Analysis. In Soil enzymes. R.W. Weaver S.A., P. Bottomley, D. Bezdicek, S. Smith, A. Tabatabai and A. Wollum (eds). pp. 775-833.

Toju H., Tanabe A.S., Sato H. (2018) Network hubs in root-associated fungal metacommunities. Microbiome 6: 116.

Van der Heijden M.G., Klironomos J.N., Ursic M., Moutoglis P., Streitwolf-Engel R., Boller T. et al. (1998) Mycorrhizal fungal diversity determines plant biodiversity, ecosystem variability and productivity. Nature 396: 69-72.

Van Der Heijden M.G., Martin F.M., Selosse M.A., Sanders I.R. (2015) Mycorrhizal ecology and evolution: the past, the present, and the future. New Phytologist 205: 1406-1423. 
van der Heijden M.G.A., Hartmann M. (2016) Networking in the plant microbiome. PLoS Biology 14: e1002378.

Vandenkoornhuyse P., Quaiser A., Duhamel M., Le Van A., Dufresne A. (2015) The importance of the microbiome of the plant holobiont. New Phytologist 206: 1196-1206.

Vorholt J.A. (2012) Microbial life in the phyllosphere. Nat. Rev. Microbiol. 10: 828-840.

White T.J., Bruns T., Lee S., Taylor J. (1990) PCR Protocols. In Amplification and direct sequencing of fungal ribosomal RNA genes for phylogenetics. Innis M.A., Gelfand D.H., Sninsky J.J., White T.J. (eds). San Diego: Academic Press, pp. 315-322.

Wu D., Zhao Z., Han X., Meng F., Wu W., Zhou M. et al. (2018) Potential dual effect of nitrification inhibitor 3,4-dimethylpyrazole phosphate on nitrifier denitrification in the mitigation of peak $\mathrm{N}_{2} \mathrm{O}$ emission events in North China Plain cropping systems. Soil Biology and Biochemistry 121: 147-153.

Xiong C., Zhu Y.G., Wang J.T., Singh B.K., Han L.L., Shen J.P. et al. (2020) Host selection shapes crop microbiome assembly and network complexity. New Phytologist, in press.

Xue Y., Chen H., Yang J.R., Liu M., Huang B., Yang J. (2018) Distinct patterns and processes of abundant and rare eukaryotic plankton communities following a reservoir cyanobacterial bloom. ISME J 12: 2263-2277.

Yao H., Sun X., He C., Maitra P., Li X.C., Guo L.D. (2019) Phyllosphere epiphytic and endophytic fungal community and network structures differ in a tropical mangrove ecosystem. Microbiome 7: 57.

Yu P., Wang C., Baldauf J.A., Tai H., Gutjahr C., Hochholdinger F., Li C. (2018) Root type and soil phosphate determine the taxonomic landscape of colonizing fungi and the transcriptome of field-grown maize roots. New Phytologist 217: 1240-1253.

Zhang D. (2004) Plant life-history evolution and reproductive ecology. Beijing: Science Press 110: 303.

Zhang W., Cao G., Li X., Zhang H., Wang C., Liu Q. et al. (2016) Closing yield gaps in China by empowering smallholder farmers. Nature 537: 671-674. 
Zhao Z.B., He J.Z., Geisen S., Han L.L., Wang J.T., Shen J.P. et al. (2019) Protist communities are more sensitive to nitrogen fertilization than other microorganisms in diverse agricultural soils. Microbiome 7: 33.

Zhou J., Deng Y., Zhang P., Xue K., Liang Y., Van Nostrand J.D. et al. (2014) Stochasticity, succession, and environmental perturbations in a fluidic ecosystem. Proc. Natl. Acad. Sci. U S A 111: E836-E845.

Ziegler M., Eguiluz V.M., Duarte C.M., Voolstra C.R. (2018) Rare symbionts may contribute to the resilience of coral-algal assemblages. ISME J 12: 161-172. 


\section{Figures legends}

Fig. 1 Phylogenetic tree, taxonomic composition, and functional guilds of crop-associated mycobiomes. (a) Phylogenetic distribution of 142 abundant taxa and 5397 rare taxa. (b) Pie plot showing the community compositions and functional guilds of abundant and rare sub-communities. (c) The difference of phylogenetic diversity, beta diversity, and niche breadth between abundant and rare sub-communities. Different letters above the boxes indicate a significant difference determined by nonparametric Kruskal-Wallis test. Abundant, abundant fungal sub-community; Rare, rare fungal sub-community; Moderate, moderate fungal sub-community.

Fig. 2 Distribution patterns of crop-associated mycobiomes. (a) NMDS ordinations showing the beta-diversity of all, abundant, and rare fungal communities based on the full dataset. "N" represents the effect of compartment niche, "C" represents the effect of crop species. The significance of different factors on community dissimilarity was tested with nested PERMANOVA based on weighted UniFrac distances. (b) Alpha and beta diversity of fungal communities in different compartment niches. Different letters above the boxes indicate a significant difference determined by nonparametric Kruskal-Wallis test. All, whole fungal community; Abundant, abundant fungal sub-community; Rare, rare fungal sub-community.

Fig. 3 Potential ecological functions of crop-associated mycobiomes. (a) The correlations between fungal communities, soil physicochemical characteristics, and soil enzyme activities in rhizosphere and bulk soil. Different fungal communities (all, abundant, and rare) were related to each environmental factor and enzyme activity by Mantel tests. Edge width corresponds to the Mantel's $r$ statistic for the corresponding distance correlations, and edge color denotes the statistical significance based on 999 permutations. The proportion of the pie indicates correlation strength, with higher proportion representing higher correlation strength. Factors marked in green and red 
represent greater correlations with abundant sub-community and rare sub-community, respectively. (b) The contribution of community composition and functional guilds of different sub-communities in predicting crop yield based on random forest modeling analyses. ${ }^{*} P<0.05$, ${ }^{* *} P<0.001$. MSE, mean square error. All, whole fungal community; Abundant, abundant fungal sub-community; Rare, rare fungal sub-community.

Fig. 4 Deterministic patterns and potential sources of crop-associated mycobiomes. (a) Effects of deterministic $(|\beta N T I| \geqslant 2)$ and stochastic $(|\beta N T I|<2)$ processes in shaping crop-associated fungal communities in different compartment niches. The percentage above and below the violin plot represents the relative contribution of the deterministic processes and stochastic processes, respectively. "Deter" and "Stoch” represents the average percentage of the deterministic processes and stochastic processes in 4 plant compartment niches, respectively. (b) The Source Model of Plant Microbiome (SMPM) model showing that crop-associated mycobiomes were derived primarily from bulk soil and gradually enriched by different plant compartment niches. "U" represents the unknown source, and the thickness of lines was equivalent to the source contribution. All, whole fungal community; Abundant, abundant fungal sub-community; Rare, rare fungal sub-community.

Fig. 5 Rare taxa play a key role in fungal co-occurrence network. (a-c) The network was established by calculating correlations among abundant, rare, and moderate ZOTUs. The nodes of the network are coloured according to (a) different sub-communities, (b) ecological clusters (modules), and (c) fungal classes. The sizes of the nodes are according to the degree of connection. The edges color represents positive (green) and negative (red) correlations. (d) Comparison of node-level topological features (degree and closeness centrality) among different sub-communities. (e) Comparison of relative abundance (z-score, mean \pm SEM) of each module among different 
compartment niches. Different letters indicate a significant difference determined by nonparametric Kruskal-Wallis test.

This article is protected by copyright. All rights reserved. 


\section{Supplementary information}

\section{Supplementary figures legends}

Fig. S1 Taxonomic composition and functional guilds of crop-associated mycobiomes. (a) Relative abundance of different fungal classes in six compartment niches $(n=468)$. Classes accounting for $<$ $1 \%$ of the total reads are grouped into "Other". (b) Relative abundance of different functional guilds in six compartment niches $(n=468)$. "XC" represents site "Xuchang, Henan province”, "QJ" represents site "Qujing, Yunnan Province”. All, whole fungal community; Abundant, abundant fungal sub-community; Rare, rare fungal sub-community.

Fig. S2 Relative abundance of dominant classes in different compartment niches. Different letters above the boxes indicate a significant difference determined by nonparametric Kruskal-Wallis test.

Fig. S3 Relative abundance of fungal functional guilds in different compartment niches. Different letters above the bar plots (mean \pm SEM) indicate a significant difference determined by nonparametric Kruskal-Wallis test.

Fig. S4 Shared and specific fungal taxa among different compartment niches and crops. (a-c) Venn diagrams showing the common and exclusive fungal ZOTUs for different compartment niches and crops. All, whole fungal community; Abundant, abundant fungal sub-community; Rare, rare fungal sub-community. (d) The biomarker taxa associated with the different crops identified by LEfSe analyses (only considering leaf and root compartments).

Fig. S5 Host dominates over environment in shaping crop fungal communities. NMDS ordinations showing the beta-diversity of (a) whole fungal community, (b) abundant fungal sub-community, and (c) rare fungal sub-community in different compartment niches. The significance of different factors 
on community dissimilarity was tested with nested PERMANOVA (based on weighted UniFrac distances). “XC” represents site “Xuchang, Henan province”, “QJ” represents site “Qujing, Yunnan Province". "C" represents the variation in fungal communities explained by crop species; "S" represents the variation in fungal communities explained by site. All, whole fungal community; Abundant, abundant fungal sub-community; Rare, rare fungal sub-community.

Fig. S6 Alpha diversity of crop-associated mycobiomes. Boxplot illustrating fungal Shannon index varies between two sites. “endo" represents endosphere. "XC” represents site "Xuchang, Henan province”, “QJ” represents site “Qujing, Yunnan Province”. The significance was determined by nonparametric Kruskal-Wallis test. ns $\mathrm{P}>0.05$, ${ }^{* * *} \mathrm{P}<0.001$, $* * * * \mathrm{P}<0.0001$.

Fig. S7 Potential sources of fungal community in each crop. The Source Model of Plant Microbiome (SMPM) model based on fungal source-tracking analysis showing the potential sources of fungal communities in (a) maize, (b) wheat, and (c) barley. "U" represents the unknown source, and the thickness of lines are equivalent to the source contribution. All, whole fungal community; Abundant, abundant fungal sub-community; Rare, rare fungal sub-community.

Fig. S8 Enrichment processes of crop-associated mycobiomes. (a) MA plot illustrating the enrichment and depletion patterns of the crop-associated mycobiomes in each compartment niche compared with bulk soil. Each point represents a single ZOTU (RA > 0.1\%). Each red point represents an individual enriched ZOTU, and green point represents an individual depleted ZOTU. The position along the y-axis represents the abundance fold change compared with bulk soil, and X-axis reports average ZOTU abundance (as count per million, CPM). “All” represents the numbers of the total ZOTUs (RA $>0.1 \%$ ) in each compartment niche. "A" represents abundant taxa, and "R" represents rare taxa. "DI" represents Depleted index and "DSI" represents 
Dissimilarity index. (b) Venn diagrams showing the shared and specific fungal ZOTUs in different compartment niches within the significant enriched ZOTUs and depleted ZOTUs. For those enriched and depleted ZOTUs commonly present in 4 plant compartment niches, only the top 3 taxonomy were shown.

Fig. S9 Relative abundance of group "Plant Pathogen” under different fertilization practices within each compartment niche. Different small letters above the bar plots (mean \pm SEM) indicate a significant difference determined by nonparametric Kruskal-Wallis test, and different capital letters above the bar plots indicate a significant difference based on t test. "endo" represents endosphere. "XC” represents site "Xuchang, Henan province”, "QJ" represents site "Qujing, Yunnan Province”. All, whole fungal community; Abundant, abundant fungal sub-community; Rare, rare fungal sub-community.

Fig. S10 Relative abundance of group “Arbuscular Mycorrhizal” under different fertilization practices within each compartment niche. Different letters above the bar plots (mean \pm SEM) indicate a significant difference determined by nonparametric Kruskal-Wallis test. "endo" represents endosphere. “XC” represents site “Xuchang, Henan province”, “QJ” represents site “Qujing, Yunnan Province”. All, whole fungal community; Abundant, abundant fungal sub-community; Rare, rare fungal sub-community. 


\section{Supplementary tables legends}

Table S1 The number of ZOTU and sequences of all, abundant, and rare communities in different compartment niches

Table S2 Effects of compartment niche, crop species, site, and fertilization practice on the fungal communities

Table S3 Spearman's correlations between fungal communities, soil physicochemical characteristics, and soil enzyme activities based on Mantel tests

Table S4 Core taxa of fungal community in different compartment niches

Table S5 Effects of compartment niche, crop species, site, and fertilization practice on the functional guilds of fungal communities 
a $\mathrm{P}$-value $-\mathrm{P}<=0.001-0.001<\mathrm{P}<=0.01-0.01<\mathrm{P}<=0.05-\mathrm{P}>0.05$ Mantel's $\mathrm{r} \longrightarrow 0.7=0.5-0.7-0.25-0.5-0.25$

SR: Soil respiration

NA: Nitrogenase activity

CB: $\beta$-D-cellobiosidase

AG: $\alpha$-glucosidase

$X Y L: \beta$-xylosidase

PNR: Potential Nitrification Rate

BG: $\beta$-glucosidase

DEA: Denitrifying enzyme activity

NAG: $\beta$-N-acetyl-glucosaminidase

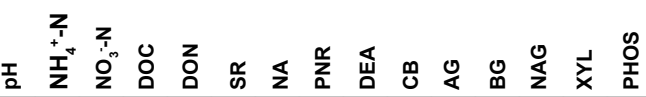

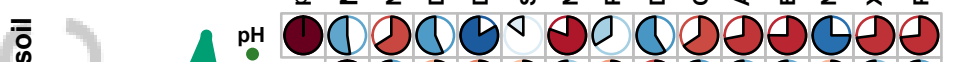

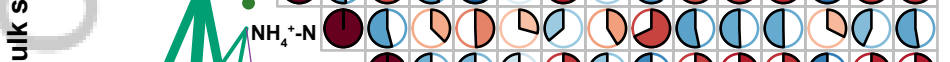

$\overline{\bar{n}}$

五

All

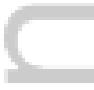

Abundant
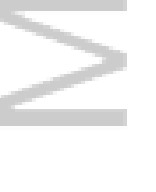

(1)

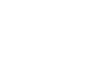

Rare

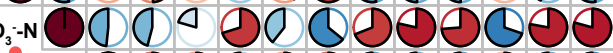
Doc ODOC DOOOOOOO DON 1 ' SR na OO ODODODOO pnr ODO DO DOO - dea OQ o O DOQ CB $A G$ DOMOC OOOCO BG NAG

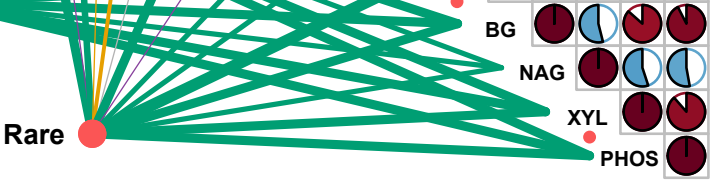

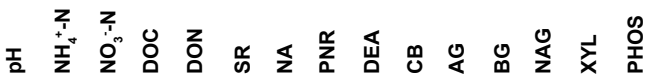
$\overline{0}$
o
0
$\frac{0}{0}$
$\frac{1}{0}$
$\frac{0}{0}$
$\frac{N}{2}$
$\bar{c}$

All

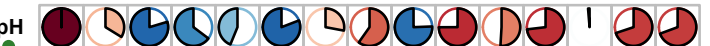

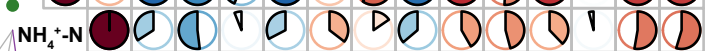

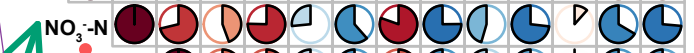

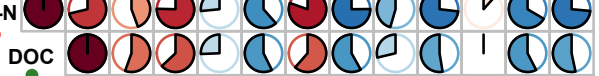

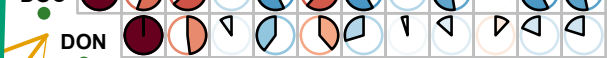
SR OOOCOOQ DOO na Do DOD D DOD i pnr OQD Do DOD - dea OQOO DQS cв OOO DO

Abundant

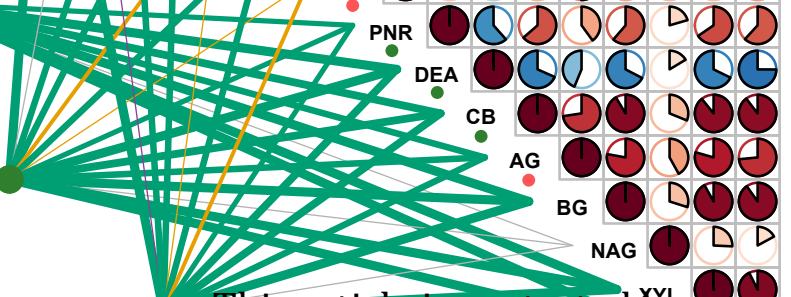

Rare
PHOS: Phosphatase

All Abundant Rare

Community composition + Functional guilds

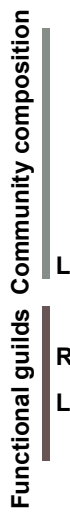

Rhizoplane -

Phylloplane -

Phylloplane -

Rhizoplane -

Rhizoplane -

Leaf endosphere -

Phylloplane -

Rhizosphere soil -

Leaf endosphere -

Phylloplane -

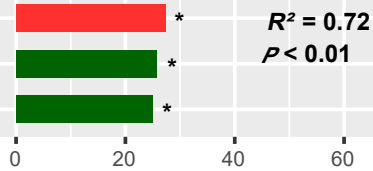

Community composition

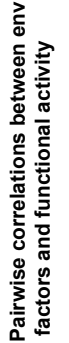

Leaf endosphere -

Rhizoplane -

Phylloplane -

Rhizoplane -

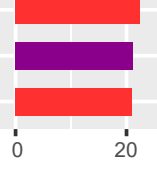

$$
R^{2}=0.72
$$$$
P<0.01
$$

\section{Functional guilds}

Rhizosphere soil -

Rhizosphere soil -

Phylloplane -

Phylloplane -

Root endosphere PHOS

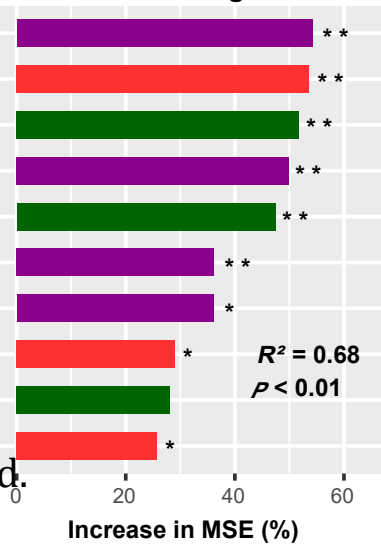




\section{a Niche Bulk soil Rhizosphere soil Rhizoplane Phylloplane Root endosphere Leaf endosphere}

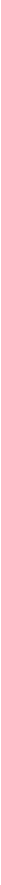


b - 0000000 - 000800000 - 9200000 a 000 - 00020000000000 - o 09000000000 - o o 01000000

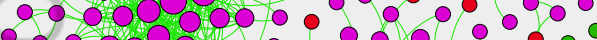
- 000800 o a a 0000

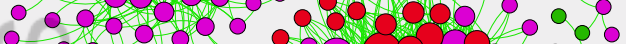
0000000.08200 - 0000000 000100000 hly? 00000000000

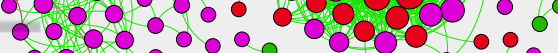

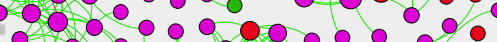
Jo. $00000 \%$

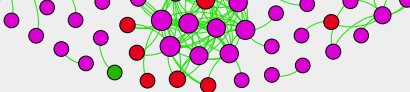
Node: 345 Edge: 2013

Abundant taxa $(8.12 \%) \quad$ Moderate taxa $(71.30 \%)$ Rare taxa $(20.58 \%)$ Degree $\bullet \bullet$

Co-occurrence (99.1\%) — Mutual exclusion (0.9\%)

d Abundant taxa Moderate taxa d Rare taxa

$p=7.4 \mathrm{e}-5$

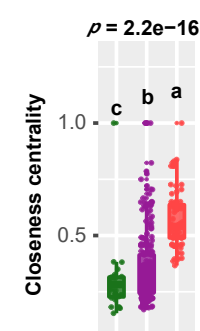

$0.0 \cdot$

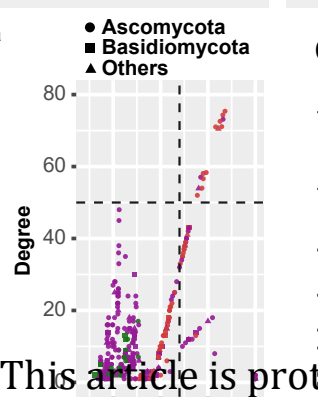

This atetel'e is prot
Mod \#10

- 00000000 .

- 010000090 - o

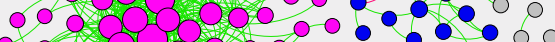

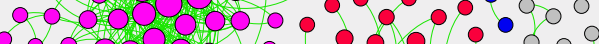

- 00090000.08900

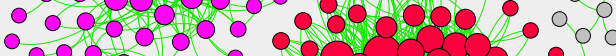

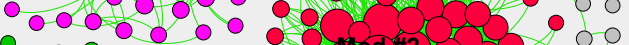

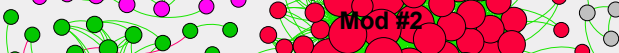

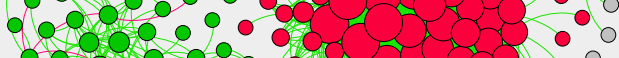

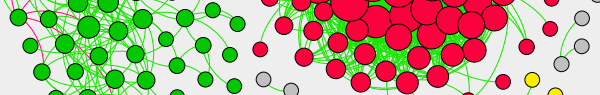

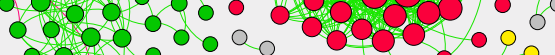

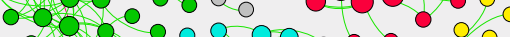

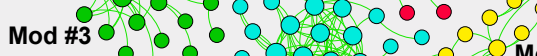

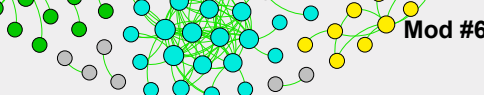

$$
\begin{aligned}
& \text { Mod \#4 }
\end{aligned}
$$

Module \#1 (28.41\%) Module \#2 (27.25\%)

Module \#3 (15.65\%) Module \#4 (7.25\%)

Module \#5 (6.67\%) Module \#6 (3.48\%)

Other modules (11.29\%)

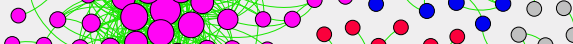

C

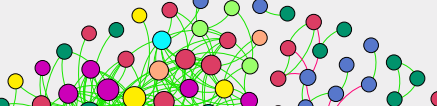
- 1090000 a o o o

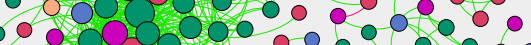

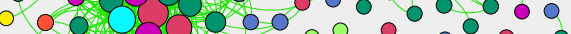

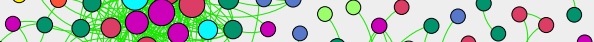

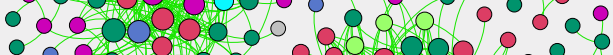
- o 000000000000

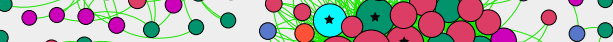

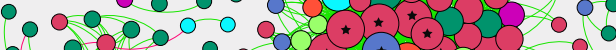

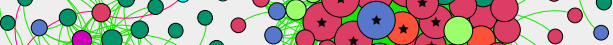

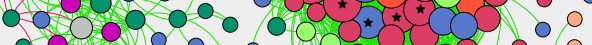
- 0020 o 000 ono o

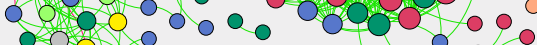
080 o o 9000000

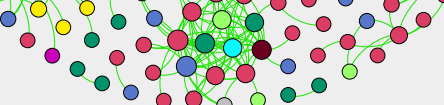

Dothideomycetes $(31.59 \%)$ Sordariomycetes $(29.57 \%)$ Unidentified (14.49\%) - Eurotiomycetes $\mathbf{( 9 . 5 7 \% )}$

Agaricomycetes $(5.22 \%)$ Mortierellomycetes $(2.90 \%)$

(2) Letiomycetes $(2.03 \%)$

Tremellomycetes $(1.74 \%)$

Lecanoromycetes $(1.16 \%)$

Others $(1.44 \%)$

$\star$ Hub species

e Niche Phylloplane Leaf endosphere Rhizoplane Root endosphere Rhizosphere soil Bulk soil

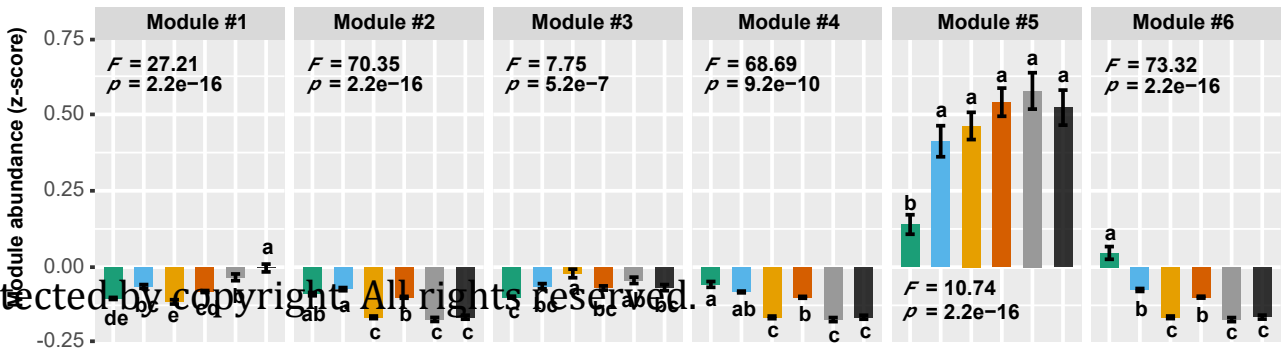




\section{University Library}

\section{$\bullet-\underset{a c c e s s}{M}$ IN E R VA}

\section{A gateway to Melbourne's research publications}

Minerva Access is the Institutional Repository of The University of Melbourne

\section{Author/s:}

Xiong, C;He, J-Z;Singh, BK;Zhu, Y-G;Wang, J-T;Li, P-P;Zhang, Q-B;Han, L-L;Shen, J-P;Ge, $\mathrm{A}-\mathrm{H} ; \mathrm{Wu}, \mathrm{C}-\mathrm{F} ; \mathrm{Zhang}, \mathrm{L}-\mathrm{M}$

Title:

Rare taxa maintain the stability of crop mycobiomes and ecosystem functions

Date:

2021-04

\section{Citation:}

Xiong, C., He, J. -Z., Singh, B. K., Zhu, Y. -G., Wang, J. -T., Li, P. -P., Zhang, Q. -B., Han, L. -L., Shen, J. -P., Ge, A. -H., Wu, C. -F. \& Zhang, L. -M. (2021). Rare taxa maintain the stability of crop mycobiomes and ecosystem functions. Environmental Microbiology, 23 (4), pp.1907-1924. https://doi.org/10.1111/1462-2920.15262.

Persistent Link:

http://hdl.handle.net/11343/276428 